\title{
Mothers Against Decapentaplegic Homolog 1
}

National Cancer Institute

\section{Source}

National Cancer Institute. Mothers Against Decapentaplegic Homolog 1. NCI Thesaurus. Code $C 106613$.

Mothers against decapentaplegic homolog 1 (465 aa, $52 \mathrm{kDa}$ ) is encoded by the human SMAD1 gene. This protein plays a role in regulating the action of bone morphogenic proteins in cell growth, apoptosis, morphogenesis, development and immune responses. 